\title{
On the Characterization of Variable Reliability Transport Protocol for Communication with Robotic Sensor Set-up
}

\author{
Debanik Roy \\ Division of Remote Handling \& Robotics, Bhabha Atomic Research Centre and Homi Bhabha National Institute, Department of Atomic \\ Energy, Governmant of India, Mumbai, India
}

Email address:

deroy@barc.gov.in

\section{To cite this article:}

Debanik Roy. On the Characterization of Variable Reliability Transport Protocol for Communication with Robotic Sensor Set-up. International Journal of Science and Qualitative Analysis. Vol. 5, No. 2, 2019, pp. 36-42. doi: 10.11648/j.ijsqa.20190502.12

Received: April 18, 2019; Accepted: August 20, 2019; Published: September 4, 2019

\begin{abstract}
Performance of bi-directional communication protocols, working under networked routing, is significantly dependent on the amount of reliability they offer during a data transmission session. A novel transport-layer protocol, namely, Variable Reliability Dataflow Protocol (VRDP) was earlier developed by the author that successfully incorporated customization, so far as selecting a desired level of reliability was concerned. However, it addressed only the generic lemma of the protocol, barring its actual application potentials. In this paper, we propose a refined enhanced version of erstwhile VRDP, wherein specific focus has been brought in towards activation and/or information retrieval of/from a robotized system. The currently developed architecture, namely, Variable Reliability Transport Protocol, is in a way more transparent from end-use point of view and is an advanced top-up over the earlier protocol. This paper delineates the algorithmic lemma, functional paradigms, mathematical model and characterization of the developed protocol and presents its performance benchmarking with the existing protocols, vide TCP \& UDP, vetted by field - trials with a robotic sensor.
\end{abstract}

Keywords: Characterization, Transport Layer, Reliability, Network, Robotic Systems, Modeling

\section{Introduction}

Algorithmic development of the transport protocol has been focused mainly on the output-parameter driven approach, barring the inherent characterization of the same. Thus, benchmarking a new protocol has been hitherto done with respect to standard metrics only like number of data packets sent / received, packet-loss, signal-to-noise ratio (SNR) etc. However, with the advent of computational tool as well as diagnostic facets, researchers have paid due attention towards unfolding some of the in-built structural features of the protocol at its algorithm level. One such primary estimate is the characterization of a new protocol by transmission reliability, which was developed by the research-team of the author [1]. In the current work, the said conceptual framework is further substantiated through a fully-grown architecture. This has been re-christened as Variable Reliability Transport Protocol (VRTP), incorporatingadvanced mathematical lemma as well as enhanced topology, culminating in real-life experimental evidence, aided by a robotic sensory system.
We generally differentiate between various network-based protocols by the degree (qualitative level) of reliability those do offer during a communication session. Until now, two extreme thresholds, viz. ('fully-reliable') Transmission Control Protocol (TCP) versus ('less-reliable') User Datagram Protocol (UDP), have been used in this scenario. The thematic distance between the two remains a void; which entails the entire gamut asopen research problem. This becomes more acute specifically in situations wherein we need to have activation and/or information retrieval of/from a robotized system. A novel transport-layer protocol, viz. VRDP, was first postulated as an interjection between TCP \& UDP [2]. The beta version of VRDP was tested successfully in LAN/WAN environment; although the need was felt that time in order to build up a more robust, tailor-made and end-use oriented professional protocol that can be readily deployable to robotic systems. The present protocol, VRTP, has been developed with full-fledged realtime application semantics, customized to robotic devices in a transparent and more user-friendly way. It is needless to mention that on-line in-situ transfer of text / image/ animation files are amply vital for robotic systems, as those must be 
communicated over the Internet with minimum loss of datapacket. We have to be extra careful, especially in data communication in reverse direction, i.e. from the robotic device to the client computer, wherein user cannot afford missing any data at all. Any loss of data-packets in such transmission would mean erroneous interpretation of the system output, e.g. sensor signals or activation-log of joint motions of the manipulator. VRTP is aimed to tackle these demanding situations, to make the outcome from the robotic units error-free, in contrast to existing protocols. However, unlike VRDP [2-4], here in VRTP, we will adhere to only two levels of parameterization, namely, VRTP-topprel and VRTPbotrel, respectively signifying VRTP with a higher \& lower level of transmission reliability.

Although the performance of TCP in an end-to-end control transfer situation has been certified to a satisfactory extent [5], its manoeuvrability in network congestion is still a pervasive bottleneck [6]. As a matter of fact, TCP proves to be more effective in text-based data transmission, which has little significance in graphics/media-based real-time communication. But, in majority of the end-applications using robotic systems, e.g. mobile units, sensors, endeffectors or even the joint motions of the manipulator, graphic-based data files are inevitable. And, TCP is fast losing edge in this regard, although the performance of TCP/IP for networks with higher bandwidth has been satisfactory [7]. Nonetheless, this lack of versatility of TCP in dealing with various data-packets has attenuated the need for more customized protocol(s), which can take care all sorts of data files, e.g. text, graphics or mixed and that too, under various states of coherence (like synchronization). With the growth in internet access services it has become crucial to evaluate the performance of TCP/IP over systems wherein bottlenecks like speed on the path carrying the acknowledgement is considerably slower than that on the forward path [8]. In our earlier works [9 -11], we first put forward a novel transport-layer server-free protocol towards maneuvering mechatronic systems, which basically tackles the peer-to-peer communication in a distributed architecture. Our protocol was found effective in overcoming a number of teething troubles in networked transmission, which are quite prudent with TCP/UDP-driven communication. The core paradigm therein was further refined and pruned for robotic applications by us, by way of sophistication of the primary protocol in [12-13].

The use of specific protocol for image-based file transfer vis-à-vis communication [14] is significant in some cases but it performs poorly so far as reliability of communication is concerned. Recent researches in developing new protocols using some modified versions of TCP are centered mostly on the issue of "acknowledgement" codes [15], but apparently reliability aspect is ignored hitherto. Nonetheless, an optimally efficient semantics towards scheduling of datapackets for internet-based communication in near future cannot afford to be less reliable [16], irrespective of the protocols used [17-20], including that of in FTP serer [21].And, with this count of built-in reliability for image- induced data-files, our protocol can prove to be advantageous. Nonetheless, we have observed that a stream of text-graphics combined file works pretty satisfactorily once we are able to switch between different modulations of a protocol, based on variable levels of transmission reliability $[1,22]$.

The saga of research towards development of 'reliable' multi-layer network protocol gained momentum in the past decade, chronologically initiated with building secure \& reliable covert channel [23] and cyber-physical adaptive protocol [24], till we have achieved adaptive protocol with variable timer [25]. No doubt, ensemble system reliability of the networked protocol suffers under multiple communication channels [26] and/or for systems exposed to network congestion [27]. Nonetheless, reliability of a transmission protocol, working under wireless network or dataless device needs subtle investigation on the parameters like sampling frequency and congestion of various real-time sensors of the network [28-30].

The paper has been organized in six sections. Details of the modeling thematic of the new protocol have been discussed in the next section. A brief outline on the characterization of the protocol is reported in section 3, followed by the algorithmic development of the logistic lemma on the protocol in section 4 . Experimental results on a real-life data transmission syntax from a robotic sensory system are reported in section 5 and finally, section 6 concludes the paper.

\section{Design Thematic of the Protocol: Model}

The developed protocol, VRTP has been modeled considering a set of watch-dog parameters, in order to suit the end-use in an optimal way.These parameters can be entrusted to work as the foundation of the system and form the necessary logistics for the protocol. The core logistic parameters are: a] number of data-packets transmitted; b] percentage of time delay intransmission; c] time of transmission; d] time of transfer and e] probability of packet loss. We will investigate the protocol-system, in light of these parameters.

\subsection{Modeling Transmission Delay}

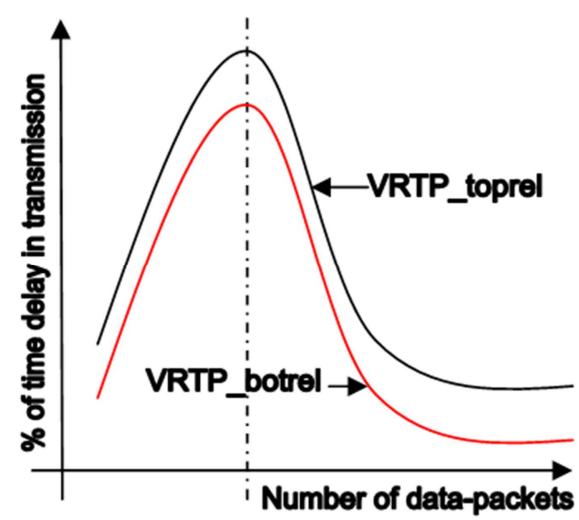

Figure 1. Variation of transmission delay versus number of data-packets. 
Transmission delay is an important parameter in judging the performance of any new network protocol. Figure 1 illustrates a schematic plot of the variation of the time delay in transmission (expressed in percentage) with respect to the number of data-packets being sent.

The plot ideally shows that the variation is relatively more severe, while transmission using VRTP_toprel, as the level of reliability is high in that protocol. Since the delay is inversely proportional to reliability, it is natural to have plot with high top-crust for VRTP_toprel, rather than VRTP_botrel. Obviously, delay attains its 'peak' approximately around the middle of the data-packet stream, and it slows down substantially at the end of the transmission. Similarly, at the beginning of the communication, we observe a comparatively low level of time delay.

\subsection{Effect of Congestion During Transmission}

Congestion control during bi-directional communication is a vital aspect of networked transmission, irrespective of the protocol used. Time delay gets fluctuated significantly under situations of uniform congestion and non-uniform congestion, with respect to finite time of transmission. The nature of this variation is independent of the VRTP-types, viz. VRTP_toprel \& VRTP_botrel. Figure 2 schematically shows the variation of the $\%$ time delay with transmission time under uniform congestion. Here, the comparative plots have been illustrated, for a specific VRTP, e.g. VRTP-i, but at two distinct time-strata, namely at $(\mathrm{t})$ and $\left(\mathrm{t}^{*}\right)$. As evident from the graph, time-strata $(t)$ is facing more congestion traffic.

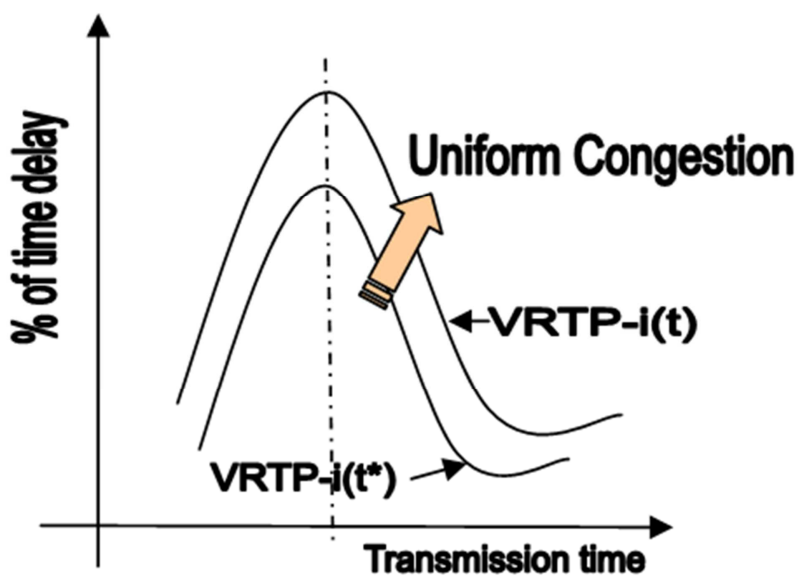

Figure 2. Plot of percentage time delay versus transmission time under uniform congestion situation using VRTP.

The situation gets significantly altered under congestion traffic that is non-uniform in nature. The variation in such case is a bit randomized; as we can't predict the network traffic load in such situations a-priori. Figure 3 schematically illustrates the variation of the $\%$ time delay with transmission time under non-uniform traffic congestion. Like earlier, here too the comparative plots have been illustrated, for a specific VRTP, e.g. VRTP-i, but at two distinct time-strata, namely at (t) and $\left(\mathrm{t}^{*}\right)$. As evident from the graph, although time-strata (t) is facing more congestion traffic, but the variation is not uniform over the phase of the transmission. Unlike the case with uniform congestion, in this case we can even think of a good optimization in selecting the VRTP-level to be used as the protocol.

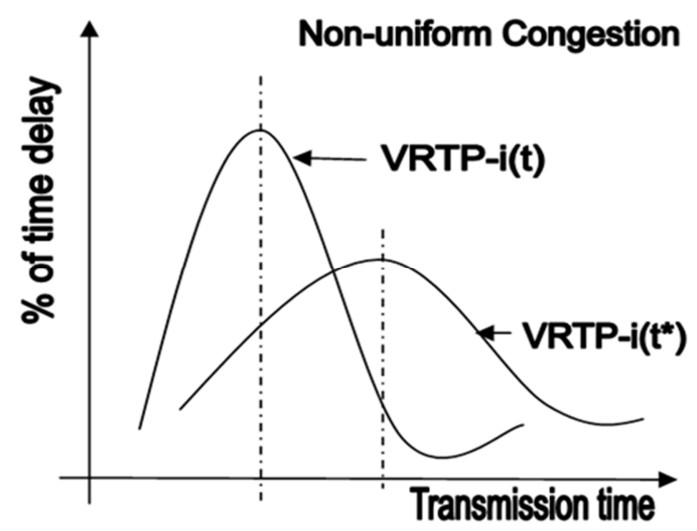

Figure 3. Variation of percentage time delay with transmission time under non-uniform congestion situation using VRTP.

\section{Characterization of the Protocol Through Analytical Paradigm}

We shall now focus on the characterization of the parametric variation of the transmission -related paradigms, pertaining to VRTP. The prime-most significant parameter to be studied under a congested network traffic is time oftransfer of data-files between the server (source) \& the client (receiver). A typical variation of the time of transfer versus the percentage of progress of transmission is plotted in figure 4.

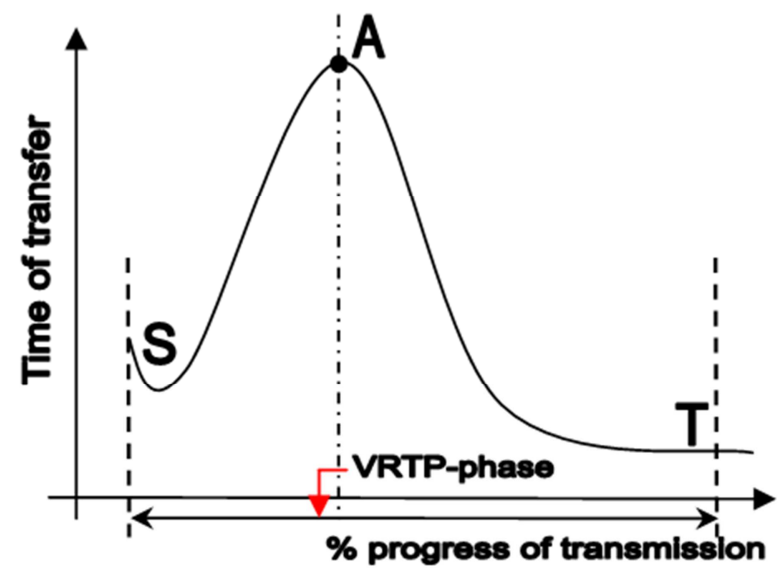

Figure 4. Characteristic curve for time of transfer under VRTP.

The curve represents the characteristic nature of the variation, over the entire phase of the operation of the protocol, namely, VRTP. This has been demarcated in the figure by ' $\mathrm{S}$ ' \& ' $\mathrm{T}$ ', respectively the start and termination of the transmission. The domain, bounded by ' $\mathrm{S}$ ' \& ' $\mathrm{T}$ ' signifies the 'VRTP-phase'.Time of transfer attains the highest, at point ' $A$ ', which essentially corresponds to the peak of the network traffic load on the system. In fact, the congestion is also the maximum around ' $\mathrm{A}$ ' and the percentage progress of 
transmission suffers in that region, irrespective of the level of VRTP.

The trade-off between two levels of VRTP, under a congested traffic load, is one interesting feature to be analyzed. Figure 5 illustrates such a situation, wherein we can have a suitable trade-off between any two levels of VRTP. This can be accomplished by switching from one level of VRTP to another during the course of transmission. As it is evident from figure 5, two characteristic curves have been plotted, respectively for two levels of VRTP. The curves represent the variation of the probability of packet-loss with respect to transmission time. While the phase of transmission in bounded between the limits ' $L$ ' \& ' $\mathrm{L}^{*}$ ', the optimal transmission will ideally follow the lower congestion path all the time. Thus, the trade-off point, 'Q' will be a vital node for the switch-over. The final pathway of the data-packets will be: i] $(A \rightarrow Q)$, and then ii] $(\mathrm{Q} \rightarrow \mathrm{B})$, both segments via the less congested paths (having comparatively lower level of probability of packet loss). However, these two segments belong to two different VRTP levels. Obviously, as indicated in the plot, the narrower curve is having the maximum congestion zone, which can be avoided by selective switching option, provided by the VRTP.

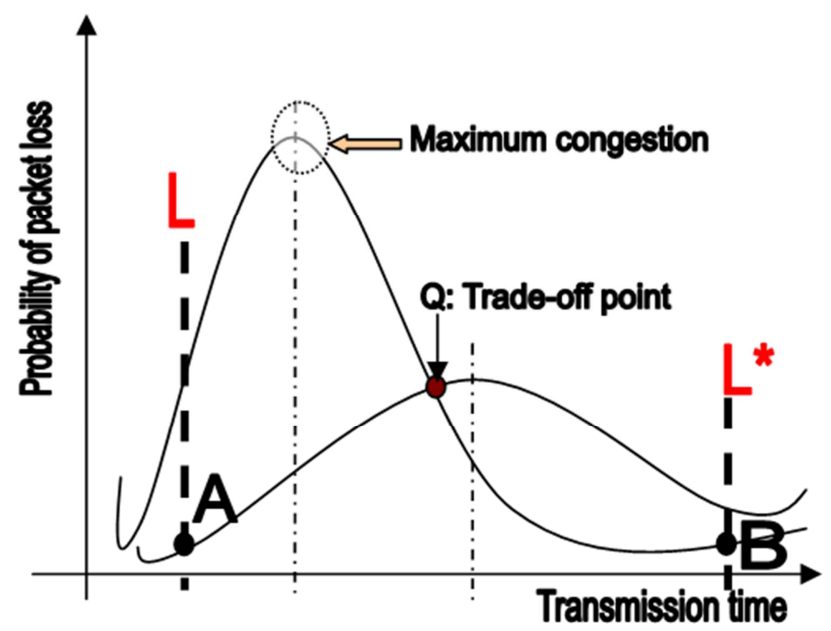

Figure 5. Trade-off for optimal pathway in transmission under congested traffic.

\section{Logistic Lemma of the Protocol Developed}

We have followed nearly the same logistic paradigm for implementing VRTP code, as we did earlier for VRDP [22], using the open-source Linux 2.4.17 kernel. Figure 6(a)schematically shows the Kernel-level customization for developing the program codes of VRTP, while the lemma for selecting the header logic is illustrated in figure6(b). The customized kernel will have handshaking with BSD socket layer and INET socket layer, having two categorized submodules (kernel), viz, 'A'\& 'B', corresponding to VRTPtoprel and VRTP-botrel. It may be mentioned here that both these two types of kernels use a tailor-made socket structure, in order to invoke seamless connection for VRTP. As VRTP essentially addresses the issue of re-transmission of packets (in case of eventual error in transport and/or loss of data packets), the header logic is based on a paradigm, namely, Dummy Segment. In this methodology, the total length of the data packet will become the summation of starting sequence number (SSN) of the dummy segment and the length of the packet (PL), followed by a Stop Bit (SB).

Unlike our work on VRDP [3-4, 22], in the present research on developing the advanced protocol VRTP, we have mainly focused on the issues concerning the retransmission syntax. This very logic provides the user with relevant information about the receiver and sender of a data packet. In fact, the specific header logic incorporating dummy segment, used in VRTP, is designed to convey the starting and ending sequence numbers of a segment being retransmitted.

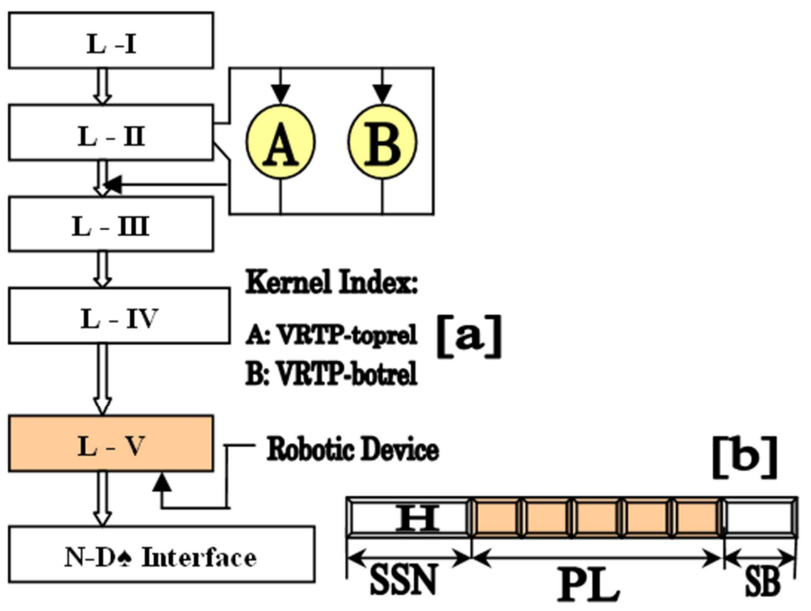

Figure 6. Schematics of VRTP: [a] Kernel Customization\& [b] Header Logic.

Index: L-I: Application Layer; L-II: BSD Socket Layer; L-III: INET Socket Layer; L-IV: Internet Protocol Layer; L-V: Hardware Layer. ^: Network Device; H: Dummy Segment Header; SSN: Starting Sequence Number; PL: Packet Length; SB: Stop Bit.

This dummy segment information is piggybacked on the next new segment to be transmitted. In general, the retransmission algorithm of VRTP is framed with a basic understanding, viz. whether it is truly needed the data-packet to be transmitted once more. The decision for re-transmission is endorsed on the basis of two attributes, namely, [i] duplication of acknowledgement and [ii] time-out in course of original transmission. The algorithm will consider both types of the VRTP options for re-transmission, followed by setting a re-transmission counter. After due addition of start and end sequence number of the VRTP option field to the header, the counter will be incremented and the re-transmission will commence thereafter. Figure 7 highlights the flow chart of the re-transmission algorithm, as used in the developed protocol. 


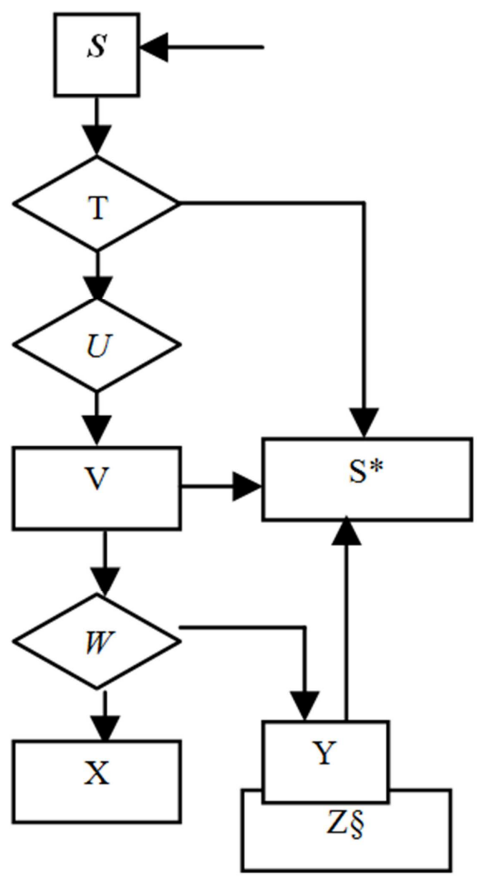

Figure 7. Flow Chart of the Re-transmission Algorithm of VRTP.

Legends: S: Start checking whether retransmission of packet is needed; T: If retransmission is required then go to ' $U$ ' else go to ' $\mathrm{S} *$ '; $\mathrm{U}$ : Check for the level of reliability and accordingly set retransmission counter value; V: Obtain counter value (initialized as counter $=\mathrm{cnt}$ ); W: If the counter $(\mathrm{cnt})$ value is greater than retransmission counter value then go to ' $\mathrm{X}$ ' else go to ' $\mathrm{Y}$ '; X: Add start and end sequence number to the VRTP header; Y: First increment counter (cnt++) and then retransmit packet; Z: Piggy back the VRTP header option to an outgoing packet and transmit; $\mathrm{S}^{*}$ : Continue usual VRTP.

$\S:$ 'Y' \& 'Z' will act simultaneously.

\section{Experimentation and Results}

The developed pair of protocol-modules of VRTPhas beenthoroughly experimented out for an effective as well as seamless reverse communication using an indigenously developed robotic sensory system. The robotic sensor is designed and developed as a tactile sensory matrix, with miniaturized struts as the sensory members. Elementary sensor-cells were located in a $3 \times 4$ rectangular array over a metallic base. Each strut is mounted with strain gauge on two opposite sides of it so that the sensor can be used both way for the detection and measurement of slip (tangential) force in plane. The sensor is activated through a dedicated PC, which performs as the system controller. This sensor controller PC is connected to a client computer, located at a remote location, separated by a physical boundary (wall). This creates an ideal environment for remote activation of the sensory system, wherein the user can receive the sensory outputs through the client computer. Figure 8 schematically shows the system layout for this experimentation. However, the data-packets from the sensor were limited to only textbased files, viz. the output signals from the external excitations (measured in milli-volts). These data were duly transferred to the remotely located client computer across the wall and finally to the daughter computers, connected to a LAN.

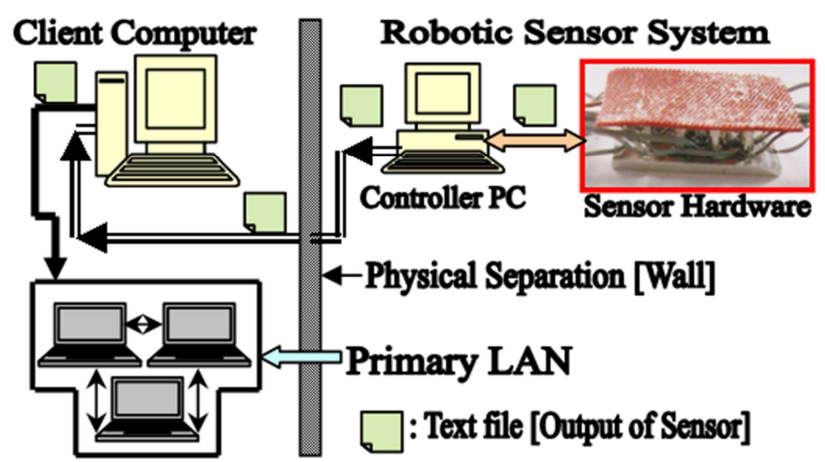

Figure 8. Schematic Layout of the Test Set-up Using Robotic Sensor System.

Experimental results have been summarized for two kinds of file type, having identical format, using two variations of VRTP as well as TCP and UDP. We used a comparatively smaller size text file (size: $48 \mathrm{~KB}$ ) to start with, while second-rung experimentation was carried out with a largersize text file (size: $850 \mathrm{~KB}$ ). However, we used the same format for all the studies pertaining to a particular set of experimentation, viz..txt for the smaller-size file and. $d o c$ for the other one.

We have tested the system performance by quantitatively observing three different distinguishing metrics, as selected for this study, namely, a] Packets received (in percentage of the total data-packets sent); b] Packet loss (in percentage of total data-packets sent) and c] Time for transfer (of the datapackets received). Table 1 presents the experimental results of the transmission of a smaller sized "Text" file (txt format). The corresponding outcome for the. doc format text file has been summarized in Table 2. We can observe that irrespective of the file-size, the performance of VRTPtoptel\&VRTP_botrel is quite steady and definitely sets new benchmark in the networked transmission.

Table 1. Transmission Anatomy of Small-size Text File Output of the Robotic Sensor Under Various Network Protocols.

\begin{tabular}{|c|c|c|c|c|}
\hline \multirow{3}{*}{$\begin{array}{l}\text { File Type } \\
\text { [Text_smaller size] } \\
\text { Size: } 48 \text { KB }\end{array}$} & \multicolumn{4}{|c|}{ Network Protocols Under Testing } \\
\hline & \multirow{2}{*}{ TCP } & \multirow{2}{*}{ UDP } & \multicolumn{2}{|l|}{ VRTP } \\
\hline & & & VRTP-toprel & VRTP-botrel \\
\hline Packets Received & All & All & All & All \\
\hline Packet Loss & $0 \%$ & $0 \%$ & $0 \%$ & $0 \%$ \\
\hline Transfer & 32.6 & 28.7 & 31.8 & 27.6 \\
\hline Time & sec. & sec. & sec. & sec. \\
\hline
\end{tabular}


Table 2. Comparison of Transmission of large-size Text File Output of the Robotic Sensor Under Different Network Protocols.

\begin{tabular}{lllll}
\hline File Type & \multicolumn{3}{l}{ Network Protocols Under Testing } & \\
\cline { 2 - 5 } [Text-larger size] & TCP & UDP & VRTP & VRTP-botrel \\
\cline { 2 - 5 } Size:850 KB & All & $841 \mathrm{~KB}$ & All & All \\
\hline Packets Received & $0 \%$ & $1 \%$ & $0 \%[$ with delay] & $0 \%$ \\
Packet Loss & 89.7 & 79.8 & 88.6 & 80.5 \\
Transfer & sec. & sec. & sec. & sec. \\
Time & & &
\end{tabular}

It may be stated here that real-time testing of a variable reliability-based networked protocol through a physical device is unique in its functionalization. In case of VRTP, we have demostrated that the performance of the protocol is moderately effective under LAN in actuating the robotic device, viz. sensory system. We have incorporated the present concept of remote activation of the robotic device (through text-based data) as a novelty and it is ready for being benchmarked. Usually, researchers have studied the performance of various network-based protocols through the metrics of bandwidth or packet loss, which are very symbolic and to some extent, theoretical measure. In contrary, our approach on performance evaluation is more direct; and, it is based on the real-life activation of a robotic system.

As a matter of fact, through this experimentation, we wish to bring out two parallel facets of networked transmission, namely: a] severity of the data-packet (type \& size) and b] complexity of the end-device / physical system. Our experiment is the basic module of these two facets sandwiched. We have successfully demonstrated the performance of the developed protocol in tackling the key modules ofboth the facets, viz. simplest data-packet (small size \& only text) and moderately low-end physocal device (sensor).

Through this case-study, we have amply demonstrated the effectiveness of VRTP in real-time field applications. It can be ascertained that barring very minor levels of transmission delay (as happened during our testing with the larger file, refer Table 2), both the variants of the new protocol take lesser time for communication compared to UDP or TCP. Nonetheless, it provides requisite reliability in transit too. And, this is the sole aspect where VRTP outperforms UDP and TCP. In our earlier work with the beta version of VRDP [3-4], it was observed that although communication through VRDP was reliable, at some levels it was taking slightly more time than TCP and/or UDP [22]. This small shortcoming was duly researched out and the present two variants of the protocol, namely, VRTP-toprel\&VRTP-botrel are successful enough in overcoming the time-lag factor, as evident from the data of Tables $1 \& 2$.

\section{Conclusions}

The commissioning of the developed protocol, VRTP, is of paramount importanceas well as of substantial advantage during networked transmission to /from a robotic system. Especially in situations like real-time simulation or graphical display-based analysis of a robotic system, such as a sensory unit, VRTP will surely provide an acceptable vis-à-vis dependable alternative to the existing protocols. In fact, VRTP will be the most desirable option because we cannot afford losing potentially important data packets or face security threats en route due to loss/faculty transmission of data packets. The need of the hour in networked robotics is to channelize more directed $R \& D$ towards real-life application of new transmission protocol and functionalize its kinetics in data transport. The saga should begin with simpler mode of data transport in form of text and gradually the transmission protocol must be able to handle data-packets with text \& graphics together. Higher-end experiments can be tried in future, on the basis of the maiden experiments, reported in the paper. The developed protocol can be entrusted with all realizable metrics that will contribute significantly towards forward \& reverse communication, as effected for a robotic device in real-time.

\section{References}

[1] Roy, D., Chatterjee, A., Jasapara, N. and Jadhav, N., "A New Transport Layer Protocol Offering Variable Reliability in Bidirectional Communication: Application For Robotic Systems", International Journal of Computers and Applications, vol. 28, no.3, August 2006, pp 251-258.

[2] Roy, D., Chatterjee, A., Jasapara, N. and Jadhav, N., "Algorithmic Development of a Novel Transport Layer Protocol Offering Variable Reliablity Towards Two-way Communication of Robotic Systems", Proceedings of the IASTED International Conference on Communications Systems and Networks ["CSN 2004"], Marbella, Spain, September 1-3, 2004.

[3] Roy, D., Chatterjee, A., Jasapara, N. and Jadhav, N, "A New Transport Layer Protocol Offering Variable Reliability: Application For Robotic Systems", Proceedings of the 2nd. IASTED International Conference on Communication and Computer Networks [“CCN 2004”], MIT, Cambridge, MA, USA, November 8-10, 2004.

[4] Roy, D., Chatterjee, A., Jasapara, N. and Jadhav, N, "A New Transport Layer Protocol Offering Variable Reliability in Bidirectional Communication: Application For Robotic Systems", Proceedings of the IASTED International Conference on Advances in Computer Science and Technology ["ACST 2004”], St. Thomas, US Virgin Islands, November 22-24, 2004.

[5] Shakkattai, S. and Kumar, A., "TCP Performance Over Endto-end Rate Control and Stochastic Available Capacity", IEEE/ACM Transactions on Networking, 9 (4), August 2001, pp 371-391. 
[6] Gustavo de Veciana, Tan-Jin Lee and Takin Konstantopoulos, "Stability and Performance Analysis of Networks Supporting Elastic Services", IEEE/ACM Transactions on Networking, Vol. 9, No. 1, Feb. 2001, pp 2-14.

[7] Lakshman, T. V., Madhow, U. and Suter, B., “ TCP/IP Performance With Random Loss and Bi-directional Congestion”, IEEE/ACM Transactions on Networking, Vol. 8, No. 5, Oct. 2000, pp 541-555.

[8] Jitendra Padhye, Victor Firoiu, Donald F. Towsley and James F. Kurose, "Modeling TCP Reno Performance: A Simple Model and Its Empirical Validation", IEEE/ACM Transactions on Networking, Vol. 8, No. 2, April 2000, pp 133-145.

[9] Roy, D., Chatterjee, A. and Venkatesh, D., "On the Development of a Bi-directional Data Communication Vis-avis Remote Activation Protocol Over the World Wide Web Using Client-server Semantics", Proceedings of the IASTED International Conference on Modeling, Identification \& Control ("MIC 2002"), Innsbruck, Austria, Feb. 18-21, 2002.

[10] Roy, D. and Chatterjee, A.,"A Distributed Generic Architecture For Bi-directional Internet-based Remote Activation Semantics Towards Manoeuvring Mechatronic Systems in Tandem", Proceedings of the IASTED International Conference on Modelling and Simulation ["MS 2003"], California, USA, Feb. 24-26, 2003.

[11] Roy, D. and Chatterjee, A., "Web-mediated Interactive Actuation of Multiple Robots Using Modified Socket Protocol", Proceedings of the 2nd. IASTED International Conference on Communication and Computer Networks ["CCN 2004"], MIT, Cambridge, MA, USA, November 8-10, 2004.

[12] Roy, D. and Chatterjee, A., "A Distributed Generic Architecture for User-interactive Internet-based Remote Activation Towards Manoeuvring Mechatronic Systems in Tandem”, Journal of Intelligent and Robotic Systems, vol. 45, no. 3, March 2006, pp 217-233.

[13] Roy, Debanik, "Control of Web-Robots Using a Customized Socket-based Protocol: A Case Study", Proceedings of the 3rd. International Conference on Informatics in Control, Automation and Robotics ["ICINCO 2006"], Setúbal, Portugal, August 2-5, 2006 [IEEE co-sponsored].

[14] Raman, S., Balakrishan, H. and Srinivasan, M., "ITP: An Image Transport Protocol for the Internet", IEEE/ACM Transactions on Networking, 10 (3), June 2002, pp 297-307.

[15] Mark, A. S. and Ramakrishnan, K. K., "Formal Specification and Verification of Safety and Performance of TCP Select Acknowledgement", IEEE/ACM Transactions on Networking, 10 (2), April 2002, pp 193-207.

[16] Xu, J. and Lipton, R. J., "On Fundamental Tradeoffs between Delay Bounds and Computational Complexity in Packet Scheduling Algorithms", IEEE/ACM Transactions on Networking, 13 (1), Feb. 2005, pp 15-28.

[17] Kalogeraki, V. and Chen, F., "Managing Distributed Objects in Peer-to-peer Systems", IEEE Network, 18 (1), Jan./Feb. 2004, pp 22-29.

[18] Sen, S. and Wang, J., "Analyzing Peer-to-peer Traffic Across Large Networks", IEEE/ACM Transactions on Networking, 12 (2), April 2004, pp 219-232.
[19] Phil Karn and Craig Partridge, "Improving Round-Trip Time Estimates in Reliable Transport Protocols", ACM Transactions on Computer Systems, vol. 9, no. 4, Nov. 1991, pp 364-373.

[20] John C -H Lin and Paul, Sanjay, "RMTP: A Reliable Multicast Transport Protocol", Proceedings of the IEEE INFOCOM,'96, 1996,

DOI: 10.1109/INFOCOM.1996.493090.

[21] Syed Mehr Ali Shah, "Measuring Round Trip Time and File Download time of FTP Servers", Network and Complex Systems, vol. 2, no. 5, 2012, pp 8-14.

[22] Roy, Debanik, "Variable Reliability Dataflow Protocol: A Novel Metric For Bi-directional Control of Mechatronic Systems", Proceedings of the IASTED International Conference on Modeling, Identification and Control, ["MIC 2006”], Lanzarote, Spain, February 6-8, 2006.

[23] Baishakhi Ray and Shivakant Mishra, "A Protocol for Building Secure and Reliable Covert Channel”, Proceedings of the IEEE $6^{\text {th. }}$ Annual Conference on Privacy, Security and Trust, 2008, DOI:10.1109/PST.2008.26.

[24] Hossein Ahmadi and Tarek Abdelzaher, "An Adaptive Reliability Cyber-Physical Transport Protocol for SpatioTemporal Data", Proceedings of the $30^{\text {th. }}$ IEEE Real-Time Systems Symposium, Dec. 1-4, 2009, U.S.A., DOI: 10.1109/RTSS.2009.45

[25] K. M. Archana Patel and Nidhi, "Sliding Window Protocols with Varaible Timer and Dynamic Buffer", Proceedings of the IEEE Internatioanl Conference on Communications and Signal Processing (ICCSP-2016), India, April 6-8, 2016, DOI: 10.1109/ICCSP.2016.7754487.

[26] Ian Foster, Jonathan Geisler, Carl Kesselman and Skrn Tuecke, "Managing Multiple Communication Methods in High-Performance Networked Computing Systems", Journal of Parallel and Distributed Computing, vol. 40, no. 1, 1997, pp $25-48$

[27] Kwon, M. and Fahmy, S., "On TCP Reaction to Explicit Congestion Notification", Journal of High Speed Networks, vol. 13, no. 2, 2004, pp 123-128.

[28] Sambheji Sarode, Kasturi Pansambal, Rupali Kshiogagnar, Anubhooti Nayakandi and Supriya Vedpather, "A survey of Transport Layer Protocols on Reliability in Wireless Sensor Networks", International Journal of Computer Science and Monile Computing, vol. 2, no. 4, 2013, pp 101-104.

[29] Arcangelo Castiglione, Raffaele Pizzolante, Bruno Carpentieri and Alfredo De Santis, "An Efficient Protocol for Reliable Data Communication on Dataless Devices", Proceedings of the 2014 IEEE International Conference on Innovative Mobile \& Internet Devices in Ubiquitous Computing, Birmingham, U.K., July 2-4, 2014, DOI:10.1109/IMIS.2014.75.

[30] Lalita Yadav and P. C. Saxena, "Challenges in Design of Transport layer Protocols for Sensor Networks", International Journal of Latest Trends in Engineering and Technology (IJLTET), vol. 2, no. 1, Jan. 2013, pp 291-294, ISSN:2278$621 X$. 